(C) 2017. This manuscript version is made available under the CC-BY-NC-ND 4.0 license. This is the Accepted Manuscript version of an article accepted for publication in JINST. IOP Publishing Ltd is not responsible for any errors or omissions in this version of the manuscript or any version derived from it. The Version of Record is available online at https://doi.org/10.1088/1748-0221/12/11/C11017 $19^{\text {TH }}$ Workshop on Radiation IMAging Detectors 2 - 6 JULY 2017 KRAKOW, POLAND

\title{
Influence of $\delta$ p-doping on the behaviour of GaAs/AIGaAs SAM-APDs for synchrotron radiation
}

\author{
T. Steinhartova ${ }^{a, b, 1}$, C. Nichetti ${ }^{b, c, d}$, M. Antonelli ${ }^{d}$, G. Cautero ${ }^{d, e}$, R. H. Menk ${ }^{d, e, f}$, \\ A. Pilotto ${ }^{c}$, F. Driussi ${ }^{c}$, P. Palestric ${ }^{c}$, L. Selmi ${ }^{c}$, K. Koshmak $^{a}$, S. Nannarone ${ }^{a}$, F. Arfelli ${ }^{b, e}$, \\ S. Dal Zilio ${ }^{a}$, G. Biasiol ${ }^{a}$ \\ ${ }^{a}$ IOM CNR, Laboratorio TASC, Area Science Park Basovizza, 34149 Trieste, Italy \\ ${ }^{b}$ Department of Physics, University of Trieste, 34128 Trieste, Italy \\ ${ }^{c}$ DPIA, University of Udine, Udine, Italy \\ ${ }^{d}$ Elettra-Sincrotrone Trieste S.C.p.A, Area Science Park Basovizza, 34149 Trieste, Italy \\ ${ }^{e}$ Istituto Nazionale di Fisica Nucleare, INFN Sezione di Trieste, Trieste, 34100, Italy \\ ${ }^{f}$ Department of Medical Imaging, University of Saskatchewan, Saskatoon, SK S7N 5A2, Canada \\ E-mail: tereza.steinhartova@phd.units.it
}

AвSTRACT: This work focuses on the development and the characterization of avalanche photodiodes with separated absorption and multiplication regions grown by molecular beam epitaxy. The i-GaAs absorption region is separated from the multiplication region by a $\delta$ p-doped layer of carbon atoms, which ensures that after applying a reverse bias, the vast majority of the potential drops in the multiplication region. Therein, thin layers of AlGaAs and GaAs alternate periodically in a socalled staircase structure to create a periodic modulation of the band gap, which under bias enables a well-defined charge multiplication and results in a low multiplication noise. The influence of the concentration of carbon atoms in the $\delta$ p-doped layer on the device characteristics was investigated and experimental data are presented together with simulation results.

KEYwords: GaAs, photodetector, avalanche photodiode

ArXiv ePrint: 1234.56789

\footnotetext{
${ }^{1}$ Corresponding author.
} 


\section{Contents}

1 Introduction 1

2 Device growth and fabrication $\quad 1$

3 Characterization in dark 3

4 Response to the light 5

5 Conclusion 6

\section{Introduction}

Avalanche photodiodes (APDs) for X-ray detection have been traditionally based on silicon, as it is nowadays the most mature and prevalent technology [1]. However, with the advent of next generation light sources more stringent requirements towards higher count rate, higher quantum efficiency for medium and high energy x-rays are put on APD detectors [2], which would benefit from a new choice of materials. In particular, $\mathrm{Al}_{\mathrm{X}} \mathrm{Ga}_{1-\mathrm{x}} \mathrm{As}$-based compounds have been suggested [3]. One of the key points of $\mathrm{GaAs}$ is the higher atomic number compared to $\mathrm{Si}$, which results in higher absorption. This renders it possible to produce devices with a thinner active region and, consequently, to achieve shorter response time and better performances of the detector.

Another important advantage of using III-V materials is the possibility to tailor the material properties, notably the bandgap at the nanoscale, by changing the stechiometry $x$ in $\mathrm{Al}_{\mathrm{x}} \mathrm{Ga}_{1-\mathrm{x}} \mathrm{As}$ alloys. In this way one of the drawbacks of using $\mathrm{Al}_{\mathrm{x}} \mathrm{Ga}_{1-\mathrm{x}} \mathrm{As}$ instead of silicon in photodetectors can be overcome; in fact, part of the multiplication noise generated by these detectors is caused by the similarity of electron and hole impact ionization coefficients in GaAs [3]. Through a periodic modulation of the bandgap profile in the multiplication region, the effective impact ionization coefficients of electrons and holes can become very different and the signal-to-noise ratio improves remarkably.

This work focuses on the development of avalanche photodiodes with separated absorption and multiplication regions (SAM-APD) [4], based on GaAs/AlGaAs and grown by Molecular Beam Epitaxy (MBE). These devices include a $\delta$ p-doping layer between the absorption and the multiplication regions in order to confine most of the electric field within the latter. In particular, this paper analyses the device behaviour in dependence on the acceptor amount inserted in such $\delta$ layer. 


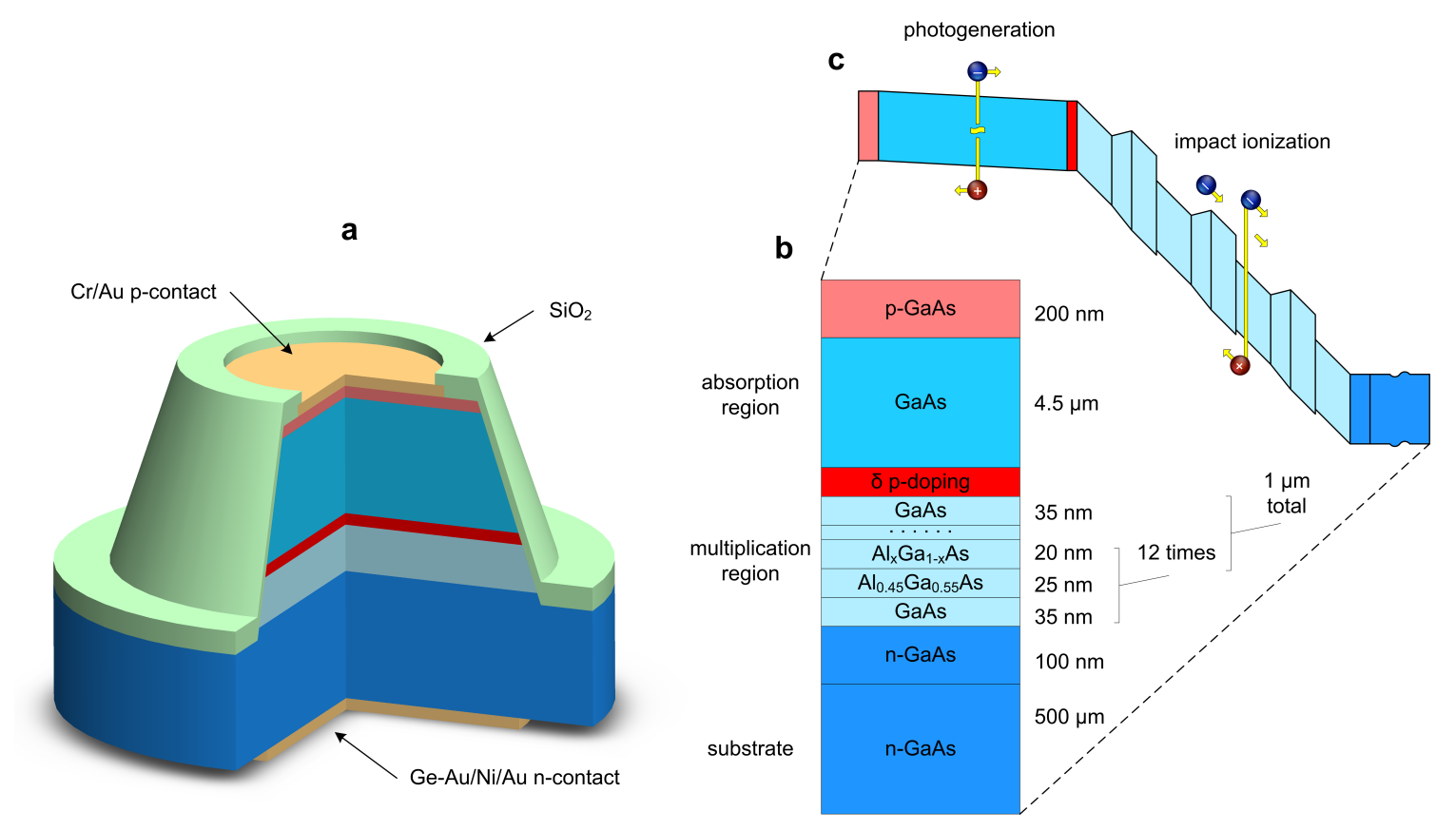

Figure 1. Sketch of the fabricated avalanche photodiode with absorption and multiplication regions.

\section{Device growth and fabrication}

The scheme of the structure grown by MBE is shown in fig. 1, b. After the deposition of a 100 -nm-thick highly n-doped $\left(2 \cdot 10^{18} \mathrm{~cm}^{-3}\right)$ buffer layer onto a (001) n-substrate, a $1 \mu \mathrm{m}$ thick intrinsic multiplication layer was grown, following the protocol of [4]. This layer includes a staircase structure with twelve stages (repetitions). Each stage consists of $35 \mathrm{~nm} \mathrm{GaAs}, 25 \mathrm{~nm} \mathrm{Al}_{0.45} \mathrm{Ga}_{0.55} \mathrm{As}$ and $20 \mathrm{~nm}$ of a linearly graded region formed by a digital alloy where the aluminum content is reduced from $45 \%$ to $1 \%$. Above the staircase structure, a 35-nm-thick GaAs spacer was grown, followed by a $\delta$ p-doping layer of carbon atoms. Such layer ensures that after applying a reverse bias the vast majority of the potential drops in the multiplication region (illustrated in fig. 1, c). On the top of the delta layer, the intrinsic $4.5-\mu \mathrm{m}$-thick GaAs absorption layer was deposited. Finally, the sample was capped with a $200 \mathrm{~nm}$ highly p-doped $\left(6 \cdot 10^{18} \mathrm{~cm}^{-3}\right)$ GaAs contact layer. The overall thickness of the active part is $5.5 \mu \mathrm{m}$. To study the influence of the $\delta$ p-doped layer on the diode performance, five samples with different carbon areal densities in such layer were grown; the values obtained by Hall-probe measurements are reported in table 1 .

Table 1. Acceptor areal densities in the $\delta$ p-doped layer as measured with Hall effect

\begin{tabular}{|c|c|c|c|c|c|}
\hline device & $\mathrm{A}$ & $\mathrm{B}$ & $\mathrm{C}$ & $\mathrm{D}$ & $\mathrm{E}$ \\
\hline density $\left[\mathrm{cm}^{-2}\right]$ & $8.8 \cdot 10^{10}$ & $5.0 \cdot 10^{11}$ & $1.3 \cdot 10^{12}$ & $1.6 \cdot 10^{12}$ & $2.5 \cdot 10^{12}$ \\
\hline
\end{tabular}

The devices were processed by photolithographic techniques. In a first step the mesa was defined by wet etching in a $\mathrm{H}_{3} \mathrm{PO}_{4}: \mathrm{H}_{2} \mathrm{O}: \mathrm{H}_{2} \mathrm{O}_{2}$ (3:50:1) solution. The structure was etched down to the $\mathrm{n}$-doped layer located beneath the multiplication region. The whole surface was then covered by $160 \mathrm{~nm}$ of $\mathrm{SiO}_{2}$ deposited by RF magnetron sputtering. The openings on the top of the mesas 
were made by reactive ion etching. The top p-contact, consisting of $10 \mathrm{~nm}$ of chromium and $50 \mathrm{~nm}$ of gold, was defined by a lift-off technique. The bottom n-contact was created by metallizing the whole back side with a 50-nm eutectic alloy of germanium-gold, $10 \mathrm{~nm}$ nickel and $40 \mathrm{~nm}$ gold. The device is sketched in fig. 1 , a. The total area of the mesas was $5 \cdot 10^{-1} \mathrm{~mm}^{2}$, while the top opening was $2.4 \cdot 10^{-1} \mathrm{~mm}^{2}$. After the fabrication, the device was mounted and bonded onto a printed-circuit carrier board provided with radio-frequency coaxial connectors, where both measurements in dark and under different light sources were performed.

\section{Characterization in dark}

The devices were first tested in dark by measuring current-voltage (IV) and capacitance-voltage (CV) characteristics. The IV measurements allow us to select devices with the lowest leakage, while the CV measurements provide information about the electric field and potential distribution inside the device. In particular, we studied the effect of the acceptor concentration in the $\delta$ p-doped layer on the CV characteristic. These measurements were performed through a precision LCR meter (HP4284A) at $1 \mathrm{MHz}$ frequency.

The left part of fig. 2 shows the capacitance per area as a function of the applied voltage, while the right part shows the depletion width calculated as

$$
d=\frac{\varepsilon_{r} \varepsilon_{0} A}{C},
$$

where $A$ is the area of the mesa, $C$ is the measured capacitance, $\varepsilon_{r}=12.6$ [5] and $\varepsilon_{0}=8.854$. $10^{-12} \mathrm{~F} / \mathrm{m}$ are the equivalent relative permittivity of the active region (calculated as the mean value of the permittivity as a function of the depletion width) and the permittivity of vacuum, respectively. It can be seen that for the highest carbon concentration in the $\delta$ p-doped layer (sample E) the capacitance is constant over the entire range of the reverse biases, up to the breakdown voltage. In these conditions, the depletion width corresponds to the thickness of the multiplication region. This means that this amount of carbon atoms is able to prevent the formation of a large electric field in the absorption region by confining the drop of the applied potential inside the multiplication region only.
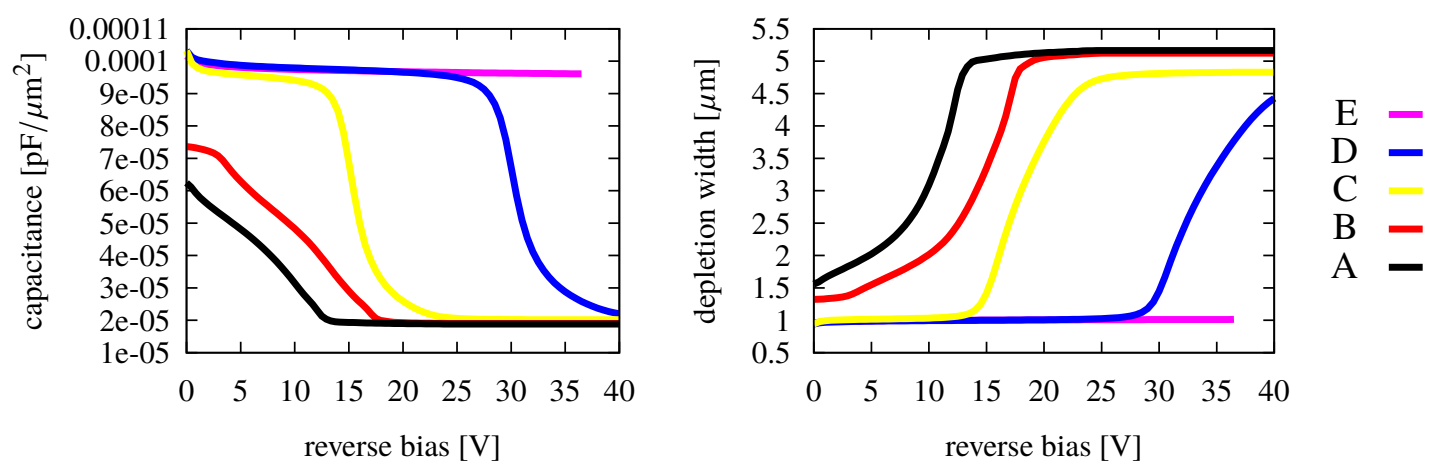

Figure 2. Capacitance and corresponding depletion width versus reverse bias for the different devices. 


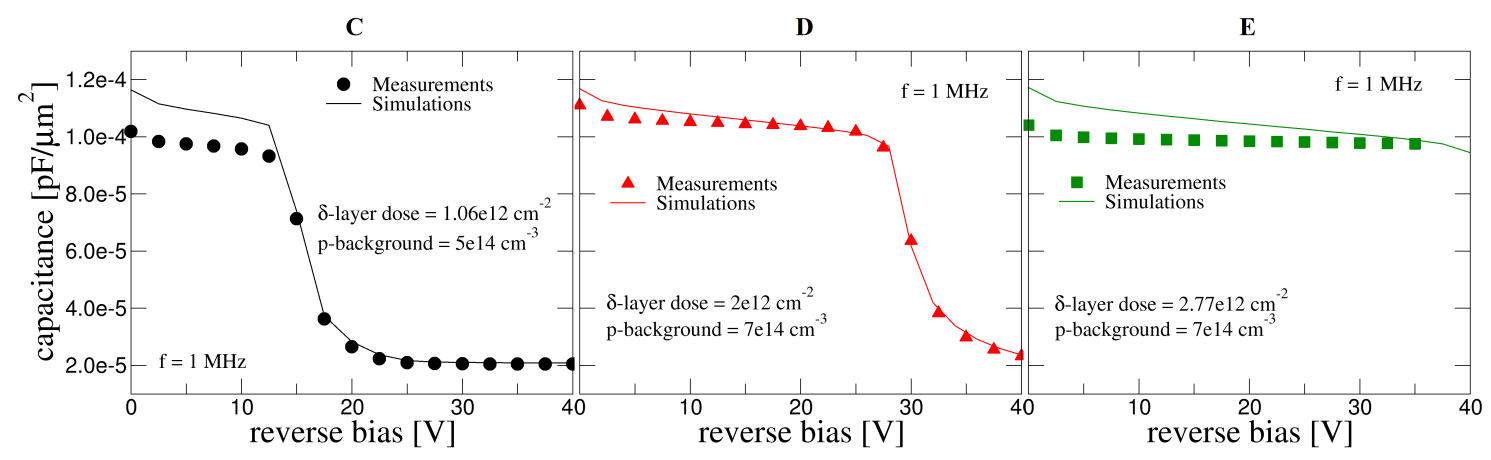

Figure 3. Comparison between experimental and simulated CV curves. In the simulation the $\delta$-layer dose is equal to the actual concentration of acceptors put in the sample, while the p-backgrounds is the residual acceptor concentration present in the absorption region.

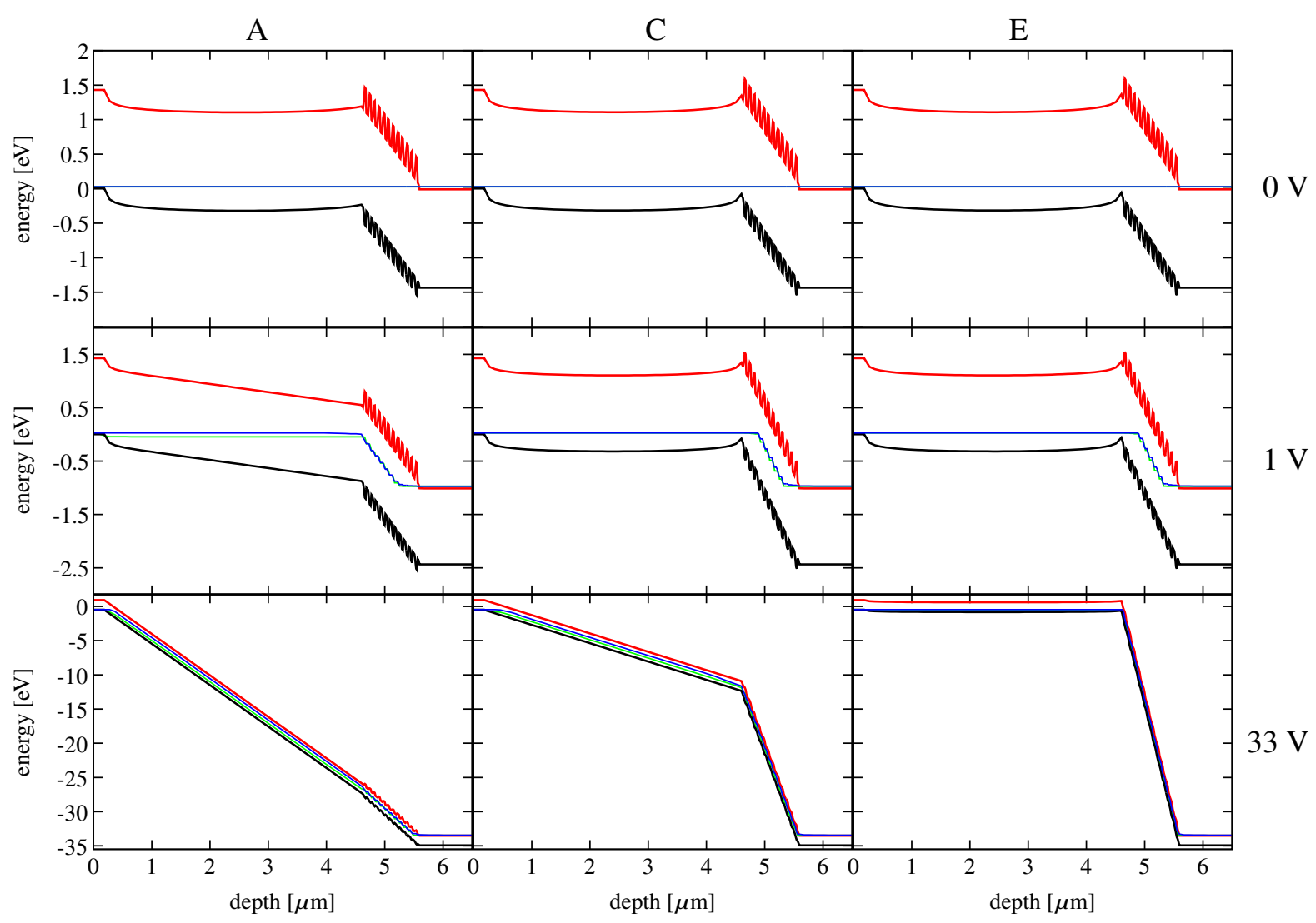

Figure 4. Band diagrams of devices A, C and E for 3 different reverse biases. Conduction band (red), valence band (black), electron and hole quasi-fermi levels (green and blue).

Conversely, in the samples with lower doping levels the capacitance drops at a certain bias, meaning that the applied potential depletes not only the multiplication region but also part of the 
absorption region. In particular, the depletion region of most of the devices with lower doping reached at some voltage a depletion width of $5.5 \mu \mathrm{m}$, which is the full thickness of the grown structure confined within the n-p contact layers. The voltage at which this drop occurs increases with the dose of the $\delta$ p-doped layer. To confirm this interpretation, we reproduced the $\mathrm{CV}$ curves of samples C, D and E with TCAD simulations [6]. Fig. 3 shows that experimental and simulated data are in good agreement; in particular, the concentration of acceptors in the $\delta$ p-doped layer obtained from simulations fairly approximates the values measured by Hall effect (tab. 1).

Fig. 4 shows the band profiles simulated at different biases with sim32 software [7] for samples $\mathrm{A}, \mathrm{C}$ and $\mathrm{E}$ to illustrate the dependence of the depleted zone on the acceptor level in the $\delta$ p-doped layer. It can be seen that for the highest doping (device E, right plots), the Fermi level at the $\delta$ layer is completely pinned to the top of the valence band at all biases, while for the lowest doping level, pinning is not complete even at zero bias, implying the creation of a potential drop in the absorption region. This explains why the $\mathrm{CV}$ characteristics (fig. 2) of the devices with low doping do not start at a capacitance value corresponding to a $1 \mu \mathrm{m}$-thick depleted zone (i.e. the multiplication region), but they are affected by the partial potential drop in the absorption region. At higher biases this effect is amplified and, at $33 \mathrm{~V}$ the absorption region is fully depleted for both samples $\mathrm{A}$ and $\mathrm{C}$. Conversely, in sample $\mathrm{E}$ depletion is confined to the multiplication region for all biases.

\section{Response to the light}

The fabricated devices were tested under light to assess their response to incoming photons. Two different light sources were used: the IOM-CNR BEAR beamline at the synchrotron Elettra (Trieste, Italy) [8] in the energy range 500-800 eV and a green $(\lambda=532 \mathrm{~nm})$ tabletop laser.

The photon energy range was selected in order to ensure that photogeneration takes place entirely within the absorption region. In fact, the absorption length is $200 \mathrm{~nm}$ and $586 \mathrm{~nm}$ at $500 \mathrm{eV}$ and $800 \mathrm{eV}$ [9], respectively, while it is equal to $140 \mathrm{~nm}$ for the laser [10]. This enables us to study the pure multiplication effect, without having it combined with charges generated in the multiplication region.

For both light sources, dark current and photocurrent were measured as a function of the applied reverse bias. The difference between these currents was calculated as the signal of the device in response to the photons. To compare the response under different light sources and different fluxes, the gain was defined as the signal normalized by its value at $20 \mathrm{~V}$, where multiplication does not take place yet:

$$
\text { gain }=\frac{\text { signal }(\text { reverse bias })}{\operatorname{signal}(20 \mathrm{~V})}
$$

Devices B, C and E were tested at the BEAR beamline. The left part of fig. 5 reports the photocurrent for different energies of the beam and the dark current of device E. The plot shows that in the experiments with higher photon energy, the photocurrent is larger. The right part of fig. 5 reports the gains calculated from the data plotted in the left part: gains for different photon energies are overlapping. This is reasonable since all the photogenerated carriers are created in the absorption region, as mentioned above, and subsequently multiplied in the multiplication region in the same way. 

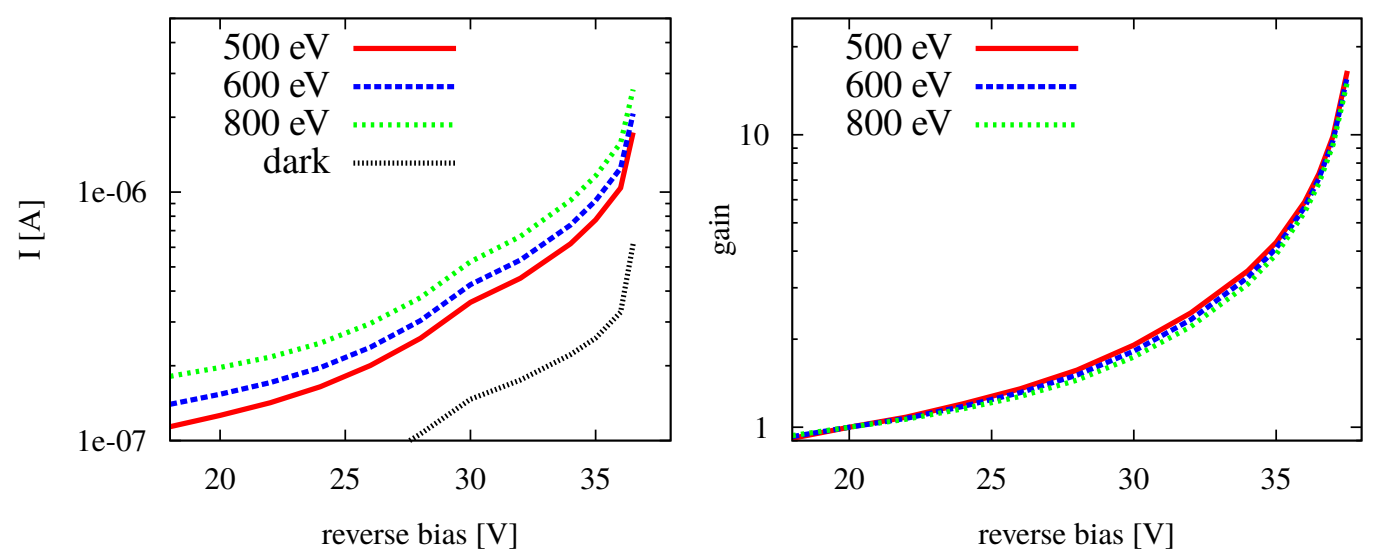

Figure 5. Dark current and photocurrent for three photon energies of the BEAR beamline in the 500-800 $\mathrm{eV}$ range of device $\mathrm{E}$ (left); gain for the same photon energies (right).
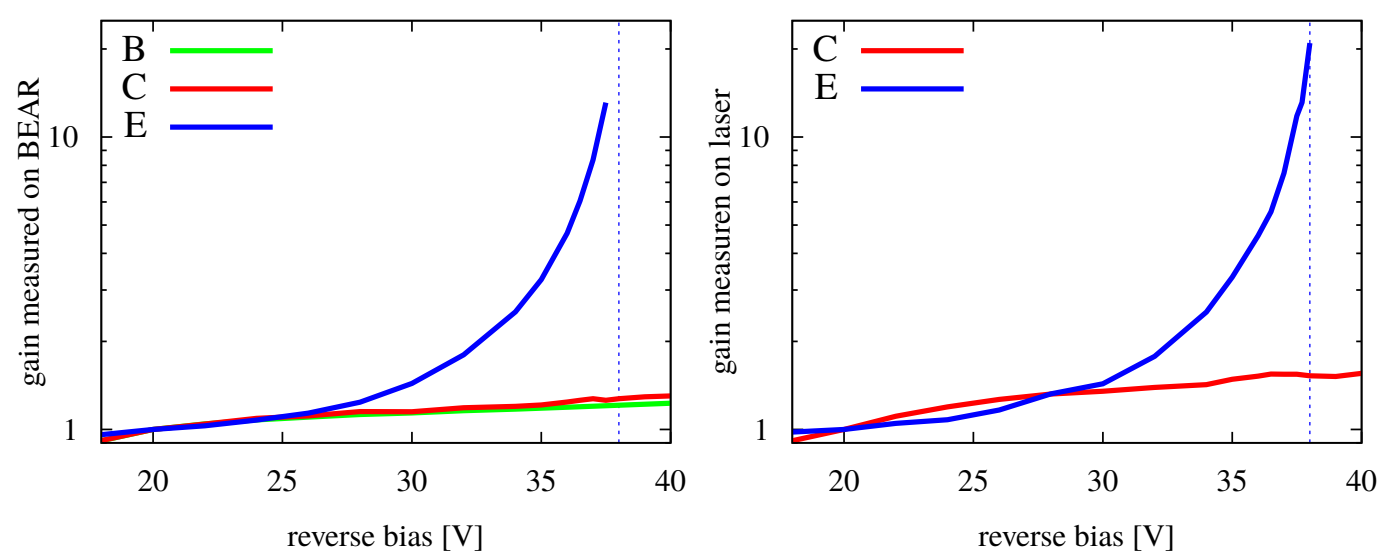

Figure 6. Gains of devices with different areal density of the $\delta \mathrm{p}$ layer measured at the BEAR beamline averaged over seven different energies in the $500-800 \mathrm{eV}$ range (left); and with the green laser (right). The brakedown voltage of device $\mathrm{E}(38 \mathrm{~V})$ is marked by a dashed line.

In the left part of fig. 6 the gains of devices B, C, E characterized on the BEAR beamline are plotted. Curves are averaged over seven different energies in the $500-800 \mathrm{eV}$ range. It can be noticed that the multiplication for the low-doping structures is minimal, while with sufficient doping it is clearly visible and reaches a value of 13.1 at $37.5 \mathrm{~V}$. The gain of the devices $\mathrm{C}$ and $\mathrm{E}$ was measured also under laser light. These data (right part of fig. 6) show the same behaviour as the one observed on the BEAR beamline, with a gain of 11.8 at $37.5 \mathrm{~V}$ and a maximum gain of 21 at $38 \mathrm{~V}$ of reverse bias. Gains measured with the two light sources coincide numerically within $10 \%$; this is due to the fact that also in the case of visible light electrons undergo the same multiplication processes as in BEAR, being generated in the first few $100 \mathrm{~nm}$ of the absorption region. 


\section{Conclusion}

Five SAM-APD samples with different areal acceptor densities in the $\delta \mathrm{p}$-doping layer separating the absorption and multiplication regions were fabricated. First, a characterization in dark was carried out and the influence of $\delta$ p-doping on the $\mathrm{CV}$ curves was observed. A dose of $2.5 \cdot 10^{12} \mathrm{~cm}^{-2}$ was found to be necessary to keep the absorption region unbiased over the whole range of reverse biases, up to the breakdown voltage. For the devices with a lower doping level, a drop in capacitance occurred at specific voltages within the measured range. Such voltage was found to decrease with decreasing doping levels. TCAD simulations are able to reproduce the measured CV curves for physical and geometrical parameter values very close to the experimental ones. The analysis of simulated potential profiles confirms the interpretation above, thus indicating the device with the highest doping as the best suited for photo multiplication applications.

The photoresponse of the devices was measured utilizing light sources, with energies low enough to ensure that the photogeneration occurs entirely within the first few $100 \mathrm{~nm}$ of the absorption region. It was noticed that for low doping levels the multiplication of generated charges is rather low (up to a gain of 2). On the other hand, when the doping level is sufficient to keep the absorption region unbiased, the whole potential difference drops within the multiplication region and a significant multiplication was observed, achieving at a reverse bias of 37.5 a gain of 11.8 under laser illumination and 13.1 in the case of the measurements performed by synchrotron light. Both these measured gains are consistent with the results published in [4]. A virtual absence of gain for lower doping levels is due to the failure to establish an adequate potential in the staircase region that would lead to electron multiplication. Our experiments underline therefore the importance of achieving a complete electrostatic separation between absorption and multiplication regions in SAM-APDs, in order to trigger a substantial avalanche effect.

\section{Acknowledgments}

The research in this work received funding by the italian MIUR through the PRIN 2015 project 2015WMZ5C8. S. Nannarone and K. Koshmak are indebted to A. Giglia for useful discussions and technical assistance.

\section{References}

[1] A. Baron, S. Kishimoto, J. Morsec and J.M. Rigalc, Silicon avalanche photodiodes for direct detection of X-rays, J. Synchr. Rad., vol. 13 (2006) pg. 131-142

[2] J. Lauter, A. Forster, H. Luth, K.D. Muller, R. Reinartz, AlGaAs/GaAs avalanche detector array-1 GBit/s X-ray receiver for timing measurements, IEEE T. Nucl. Sci., vol. 43, p. 1446-1451, 1996

[3] F. Capasso, W.T. Tsang, G.F. Williams, Staircase solid-state photomultipliers and avalanche photodiodes with enhanced ionization rates ration, IEEE T. Electron Dev., vol. 30 (1983) pg. 381-390

[4] J. Lauter, D. Protic, A. Forster, H. Luth, AlGaAs/GaAs SAM-avalanche photodiode: An X-ray detector for low energy photons, Nucl. Instrum. Meth. A, vol. 356 (1995), pg. 324-329

[5] M. E. Levinshtein, S. L. Rumyantsev, M. Shur, Handbook series on semiconductor parameters, Chapter 1, Singapore: World Scientific, 1996 
[6] Synopsys, Sentaurus ${ }^{\mathrm{TM}}$ Device User Guide, March, 2016

[7] Winston, D.W., Physical Simulation of Optoelectronic Semiconductor Devices, UCB, 1996

[8] http://www.elettra.trieste.it/elettra-beamlines/bear.html

[9] X-ray Data Booklet (January 2001) edited by A. C. Thompson, D. Vaughan

[10] E. D. Palik, Gallium Arsenide (GaAs), In Handbook of Optical Constants of Solids, Academic Press, Burlington, 1997, pg. 429-443, ISBN 9780125444156 